\section{Evaluación nutricional de personas con VIH/SIDA}

\author{
Nutritional status of people \\ living with HIV-AIDS
}

\begin{abstract}
Introduction: At present, the relationship between diet, nutrition and HIV infection has been demonstrated. The scientific community recognizes that knowledge and nutritional care can help maintain health and reduce the effects of the infection. Objective: To assess the nutritional status of people living with HIV-AIDS treated in out-patient clinics of Plaza County. Subjects and Methods: A descriptive cross-sectional study in 87 people infected with HIV (66 males and 21 females). Fifty four asymptomatic infected people and 33 AIDS patients were studied. Nutritional assessment consisted of anthropometric measurements: weight, height, triceps skinfold and arm circumference to calculate the body mass index, and fat and arm muscle area; dietary assessment with frequency questionnaires and food consumption by food groups; measurement of biochemical parameters: hemoglobin, triglycerides, blood sugar and cholesterol. Values were compared with the Student test and Spearman correlation. Results: The largest group was asymptomatic male between 30-49 years. Few cases of anemia were detected. Hypertriglyceridemia and discrete reductions in fat reserves were predominant. Half of people with AIDS presented bad eating habits. More than half presented sufficient level of knowledge and attitude. Conclusion: It is necessary to increase the attitude of these patients in relation to the care of their nutrition. Key words: nutritional assessment, HIV-AIDS, BMI, anthropometry.
\end{abstract}

Tania Massip N. (1)

Graciela Nicot B. (2)

Juliette Massip N. (3)

Asterio Valdés V. (3)

Anyelien Pimienta S. (4)

(1) Dirección Municipal de Higiene y Epidemiología de Plaza, La Habana, Cuba. (2) Instituto de Medicina del Deporte, La Habana, Cuba. (3) Hospital Universitario Calixto García, La Habana, Cuba. (4) Facultad de Ciencias Médicas Manuel Fajardo, La Habana, Cuba.

Dirigir la correspondencia a: Profesora Tania Massip Nico Dirección Municipal de Higiene y Epidemiología de Plaza. Calle San José \$1220 entre Mazón y Basarrate. Plaza. La Habana. Cuba

Teléfono: +5378701287 E-mail: gnb@infomed.sld.cu

Este trabajo fue recibido el 15 de Mayo de 2014 y aceptado para ser publicado el 5 de Marzo de 2015.

\section{INTRODUCCIÓN}

En la actualidad está demostrada la relación entre la alimentación, la nutrición y la evolución de cualquier enfermedad crónica; tal es el caso de los pacientes con la infección por VIH. La comunidad científica reconoce que los conocimientos y el cuidado nutricional pueden contribuir a mantener la salud y a disminuir los efectos de una enfermedad crónica (1).

La alimentación, aunque sea la más cotidiana, desempeña un papel importante, aportando a los pacientes portadores del virus, efectos específicos, en una situación que afecta su salud, su nutrición y los efectos secundarios de su tratamiento (2).

Una evaluación del estado nutricional completa debe incluir una evaluación clínica, mediciones antropométricas, la evaluación de los patrones alimentarios, de la ingesta de fuentes de energía y nutrientes y de algunos parámetros bioquímicos (3).

El estudio alimentación-nutrición efectuado de una forma correcta contribuye a mantener y/o mejorar la situación nutricional del paciente, a prevenir su deterioro y a aminorar los síntomas que pueden aparecer en el desarrollo de su enfermedad, obteniendo así una mejora considerable de su calidad de vida.

A menudo se han ignorado los aspectos relacionados con la nutrición cuando se trata de la infección por el VIH/ sida y se dirige la atención fundamentalmente a los tratamientos farmacológicos y a aspectos esencialmente clínicos, olvidando la importancia de la alimentación, que se pone de manifiesto desde las primeras etapas de la infección y que evita deficiencias nutricionales. Una alimentación saludable y equilibrada ayudará a mantener el peso corporal y un buen estado general (4).

El gobierno cubano les proporciona una dieta con un alto contenido de proteínas a todos las personas con VIH/ sida, para garantizarles una nutrición adecuada. Ese esfuerzo se complementa con proyectos como Nutrición y Sida del Instituto de Nutrición e Higiene de los Alimentos y el Centro 
Nacional de Prevención ITS/VIH/sida (5).

Teniendo en cuenta la información brindada se decidió realizar esta investigación con el propósito de realizar una evaluación nutricional en la población de personas con $\mathrm{VIH}$-sida (PVs) del municipio Plaza, que permitan la implementación de una serie de estrategias individuales y familiares destinadas a modificar y corregir positivamente las alteraciones que se encuentren, mediante hábitos más saludables, así como brindar información útil para establecer programas de atención en este grupo poblacional.

\section{SUJETOS Y MÉTODO}

Se realizó un estudio descriptivo, de corte transversal cuyo universo estuvo constituido por las personas diagnosticadas con VIH-sida en el municipio Plaza de la Revolución, desde el comienzo de la epidemia en 1986 hasta el año 2013.

La muestra no aleatoria estuvo conformada por las 87 personas que acudieron a la consulta de descentralización de la atención médica del municipio Plaza de la Revolución, de septiembre a diciembre del 2013 y que aceptaron participar en el estudio.

En cuanto al sexo, la relación entre masculino y femenino fue aproximadamente de 4 a 1 con un predominio del sexo masculino; de las 54 personas con $\mathrm{VIH}$ asintomáticos, sólo 12 fueron mujeres (27\%) y de los 33 del grupo con sida, 9 eran mujeres ( $22 \%)$, con un total de 21 personas del sexo femenino, $24 \%$ del total de sujetos estudiados.

Se recogieron las siguientes variables: edad, sexo, nivel de escolaridad, estadio de la enfermedad: se clasificó en portador asintomático al VIH y persona en fase sida, de acuerdo a la clasificación del CDC 1993 (6). En el grupo persona VIH asintomático o grupo A según esta clasificación se incluyó también a los del grupo B que no han presentado ninguna enfermedad oportunista marcadora de sida, dejando en el grupo $\mathrm{C}$ a las personas con sida (ya sea marcadora de sida por enfermedad o por CD4).

También se recogieron las variables: hemoglobina (OMS)7, glucosa, colesterol total y triglicéridos (National Cholesterol Education Program Adult Treatment Panel III) (8) y CD4 (CDC 1993) (6).

Se midieron las siguientes variables antropométricas:

1. Índice de masa corporal (IMC): peso $(\mathrm{Kg}) /$ estatura $\left(\mathrm{m}^{2}\right)$ James WPT y colaboradores citado por Zayas (5)

2. Circunferencia del brazo (CB) Se tomaron valores de la $C B$ de $26 \mathrm{~cm}$ en los hombres y $24 \mathrm{~cm}$ en las mujeres como puntos de corte para detectar malnutrición por defecto Según Berdasco y colaboradores (9).

3. Pliegue tricipital (PT)

4. Área Muscular del Brazo (AMB) Para malnutrición por defecto, Se fijó el criterio de nutrición de acuerdo con Berdasco y Romero (9).

5. Área Grasa del Brazo (AGB) Para la malnutrición por defecto. Se fijó el criterio de nutrición de acuerdo con Berdasco y Romero (9).

Se aplicaron las encuestas de frecuencia de comidas y de consumo por grupos de alimentos, creadas y validadas en el Instituto de Nutrición de los alimentos (INHA), para definir los hábitos de consumo de las comidas más importantes del día y de los grupos de alimentos que más predominaban en la dieta estos pacientes. En los dos casos se consideró adecuada una frecuencia de alimentación superior a 5 veces por semana, siendo no adecuada cuando el consumo era inferior a 4 veces semanales.

Se aplicó una encuesta de conocimientos de nutrición y salud, también validada en el INHA, para evaluar el nivel de conocimiento de la muestra poblacional. Su clasificación aparece en criterios para evaluar conocimientos de nutrición y salud.

Las respuestas fueron clasificadas en suficientes e insuficientes y el conocimiento individual fue suficiente cuando el número de respuestas correctas superaban el $70 \%$ del total, e insuficientes cuando fue inferior al $70 \%$.

\section{Aspectos éticos}

El Comité de Ética de la Dirección Municipal de Higiene y Epidemiología de Plaza aprobó el protocolo de investigación. Antes de ser incluido en el estudio se les solicitó a los pacientes su consentimiento informado.

\section{Análisis estadístico}

La información fue procesada con el paquete SPSS para Windows Versión 14.0. Las variables cualitativas fueron expresadas en frecuencias absolutas y relativas (porcentaje) mientras que para las cuantitativas se calculó media y desviación estándar. Se realizó comparación de medias entre estadios de la enfermedad en las variables cuantitativas utilizando la prueba t de Student para muestras independientes. Se realizó correlación de Spearman para conocer el grado de relación entre variables antropométricas y hemoquímicas con estadio de la enfermedad. Se estableció un nivel de significación de $p \leq 0,05$. Finalmente se confeccionaron tablas y gráficos que facilitaron el análisis, discusión y presentación de los resultados obtenidos.

\section{RESULTADOS}

La muestra estuvo constituida por 87 personas los cuales se clasificaron clínicamente, según el porcentaje de CD4 en sangre, en: 54 personas portadoras asintomáticos del VIH y 33 personas en fase sida, los que se mencionarán, para facilitar la exposición, como grupo asintomático y grupo sida.

En la tabla 1, considerando a cada grupo como el 100\%, se encontró que en el grupo asintomático el mayor número de personas, 51 de los 54, hasta cinco años de diagnóstico, constituyendo $94,5 \%$ de ese grupo y 5,5\% tienen más de cinco años de diagnóstico. Los períodos más prolongados de evolución de la infección correspondieron a los individuos del grupo sida (18 sujetos de un total de 33 para un 54,5\% del total de ese grupo) y sólo 15 sujetos hasta cinco años de evolución con 45,5\% del grupo. Más del 50\% de los sujetos con sida tenían una evolución mayor a los cinco años.

En la tabla 2 se presentan los valores de triglicéridos en sangre que se encuentran dentro de cifras normales en el grupo VIH y elevados en el grupo sida, encontrándose este último grupo con hipertrigliceridemia franca. Las diferencias entre ambos grupos fueron estadísticamente significativas $(p \leq 0,05)$.

$\mathrm{Al}$ analizar los resultados de los triglicéridos en ambos grupos de estudio, se encontró un porciento elevado con hipertrigliceridemia, con predominio en el grupo sida $(57,6 \%)$. En el grupo asintomático se encontró en 33,3\%, aunque menor que en el otro grupo no puede considerarse como de baja frecuencia.

En cuanto al colesterol en sangre (tabla 2), no se encontraron diferencias significativas entre ambos grupos y los valores promedios se corresponden con concentraciones de este metabolito dentro del rango normal. Llama la atención que el valor máximo para ambos grupos se corresponde con una hipercolesterolemia franca.

Se registró un porcentaje elevado de sujetos con hipercolesterolemia en ambos grupos, siendo mayor en el grupo 
asintomático que en el grupo sida. En este caso se encontró una cifra significativamente elevada de personas que presentaron niveles elevados de colesterol total.

En relación a las glicemias (tabla 2), en ambos estadios de la enfermedad los promedios de las concentraciones de este indicador se encuentran dentro de cifras normales, sin diferencias estadísticamente significativas aunque en los valores máximos encontrados para ambos grupos se observan sujetos con cifras algo elevadas.

Se encontró que un grupo importante de sujetos asintomáticos (19) tenían cifras correspondientes a hiperglicemia, así como seis de los del grupo sida. Estas personas no tenían antecedentes de hiperglicemia o diabetes mellitus.

En la tabla 3, se observa que las cifras promedio de hemoglobina se encuentran dentro de cifras normales para ambos sexos, en los del grupo asintomático. En los casos sida, en el sexo femenino discretamente por debajo de cifras normales, en tanto en el masculino dentro de cifras normales. Las diferencias entre grupos no fueron estadísticamente significativas $(p>0,05)$.

Según puntos de corte de la hemoglobina, considerándose cada grupo como el 100\%; en el grupo asintomático 92,5\% (39 sujetos) presentó cifras normales (20,3\% mujeres y $72,2 \%$ hombres), 7,4\% presentó anemia clínica (1,9\% mujeres y 5,5\%. hombres). En el grupo con sida el $87.9 \%$ (29 sujetos) presentó cifras normales (21,2\% mujeres y $66,7 \%$ hombres), el $12,2 \%$ presentó anemia clínica (6,1\% mujeres y 6,1\%. hombres).

En la tabla 4 se observa que tanto portadores asintomáticos al VIH como personas con sida presentaron valores de IMC normales en ambos sexos.

En este trabajo el valor promedio del índice de masa corporal en las personas estudiadas fue 24,01 en el grupo asintomático y valores inferiores, aunque dentro de cifras normales también $(21,44 \mathrm{Kg} / \mathrm{m} 2)$ en las personas con sida sin diferenciar por edad o sexo; sin diferencias significativas entre ambos grupos.

Al evaluar los sujetos según IMC, la mayoría era normopeso (34 del grupo asintomático y 32 del grupo sida) 63 y $96,7 \%$ del total por grupos respectivamente y 19 sujetos sobrepeso grado I, con predominio del grupo asintomático (18) (33\%). Se contó con dos personas del grupo asintomático con deficiencia energética grado I. En ninguno de los grupos

TABLA 1

Tiempo de diagnóstico de la enfermedad según clasificación clínica.

\begin{tabular}{|c|c|c|c|c|}
\hline \multirow[t]{2}{*}{ tiempo de diagnóstico } & \multicolumn{2}{|c|}{ VIH } & \multicolumn{2}{|c|}{ sida } \\
\hline & No. & $\%$ & No. & $\%$ \\
\hline 0 a 5 años & 51 & 94.5 & 18 & 45.5 \\
\hline 6 a 15 años & 3 & 5.5 & 15 & 54.5 \\
\hline Total & 54 & 100 & 33 & 100 \\
\hline
\end{tabular}

Fuente: Historia Clínica.

TABLA 2

Valores de triglicéridos, colesterol y glicemia (mmol/l) según clasificación clínica.

\begin{tabular}{|c|c|c|c|c|c|c|}
\hline \multirow[t]{2}{*}{ Clasificación clínica } & \multicolumn{2}{|c|}{ Triglicéridos } & \multicolumn{2}{|c|}{ Colesterol } & \multicolumn{2}{|c|}{ Glicemia } \\
\hline & $x$ & DS & $x$ & DS & $x$ & DS \\
\hline Asintomático & $1,44^{*}$ & 1,01 & 3,83 & 1,54 & 4,20 & 0,75 \\
\hline sida & 1,84 * & 1,33 & 4,5 & 1,13 & 4,48 & 0,68 \\
\hline
\end{tabular}

TABLA 3

Comportamiento de los niveles de Hemoglobina ( $\mathrm{g} / \mathrm{dl}$ ) según clasificación clínica y sexo.

\begin{tabular}{llcc}
\hline \multirow{2}{*}{ clasificación clínica } & & Masculino & Femenino \\
\cline { 2 - 3 } sida & Media & 13.2 & 11.5 \\
& DS & 1.1 & 1.4 \\
\multirow{2}{*}{ asintomático } & Media & 13.9 & 12.9 \\
& DS & 1.2 & 0.4 \\
\hline
\end{tabular}

Fuente: Historias clínicas. 
estudiados se encontraron individuos con deficiencia energética crónica grado II y III.

Las personas asintomáticas de este estudio se encontraron con predominio en percentiles superiores.

Al analizar, en la tabla 5 las medidas antropométricas en el sexo masculino: circunferencia braquial, pliegue tricipital, se observan valores similares (sin diferencias estadísticamente significativas) en ambos estadios de la enfermedad.

Al analizarlas en el sexo femenino: circunferencia braquial y pliegue tricipital, se observan valores similares (sin diferencias estadísticamente significativas) en ambos estadios de la enfermedad.

La mayoría de las mujeres presentaban valores normales (10 y 90 percentil). El área magra de las personas del sexo masculino del grupo asintomático, fue similar al del grupo sida, sin diferencias estadísticamente significativas. Ambos se encontraban entre los percentiles 10 y 90, según los criterios de puntos de corte para la población cubana adulta, lo que apoya que no hay depleción en este sexo de las reservas proteicas del organismo.

Los valores del área grasa en los varones en ambos estadios de la infección corresponden a valores normales, sin diferencia estadísticamente significativa entre ellos.

El área muscular del brazo en el sexo femenino se encontró dentro de valores normales para ambos estadios de la infección. Todas las personas se encontraron entre los percentiles 10 y 90 .
En cuanto al área grasa en ambos estadios las mujeres de esta investigación se encontraban por encima del percentil 10.

Al realizar estudios de correlación de indicadores antropométricos con estadio de la enfermedad en este trabajo, no se encontró, de forma estadísticamente significativa, ningún tipo de asociación entre ellos. En este estudio no se realizaron estudios de correlación con la edad.

El gráfico 1 muestra el análisis de frecuencia de comida en el día, donde $80 \%$ de las personas asintomáticas cumplieron con las seis frecuencias diarias recomendadas, 20\% tuvieron un cumplimiento no adecuado. Los mayores incumplimientos de consumo fueron en las personas asintomáticas, en la merienda de la noche, seguido por la merienda de la tarde y por último el desayuno.

En las personas con sida se encontró un número similar de sujetos con frecuencia adecuado de consumo de alimentos en el día (50\%), el otro 50\% se comportó como inadecuado. En estas personas los incumplimientos de consumo predominantes fueron la merienda de la noche, seguido por el desayuno y la comida. En relación con las meriendas, la de mayor aceptación para ambos grupos, resultó la matutina.

Como se puede observar en la tabla 6 , el consumo de los diferentes grupos de alimentos presenta un comportamiento variable, según las frecuencias. Los grupos alimentarios que más frecuentemente consumieron los pacientes de las dos categorías fueron cereales y viandas (grupo I), granos (grupo IV), los lácteos (grupo V) y las grasas de origen vegetal (grupo

\section{TABLA 4}

Evaluación de la muestra según IMC y clasificación clínica.

\begin{tabular}{|c|c|c|c|c|}
\hline \multicolumn{2}{|c|}{ Clasificación clínica } & $\begin{array}{c}\text { Sobrepeso } \\
\text { Grado I }\end{array}$ & Normopeso & $\begin{array}{c}\text { Deficiencia Energética } \\
\text { Grado I }\end{array}$ \\
\hline asintomático & $\begin{array}{c}\text { No. } \\
\%\end{array}$ & $\begin{array}{c}18 \\
33,3\end{array}$ & $\begin{array}{c}34 \\
63,0\end{array}$ & $\begin{array}{c}2 \\
3,7\end{array}$ \\
\hline sida & $\begin{array}{c}\text { No. } \\
\%\end{array}$ & $\begin{array}{c}1 \\
3,3\end{array}$ & $\begin{array}{c}32 \\
96,7\end{array}$ & $\begin{array}{l}0 \\
0\end{array}$ \\
\hline $\begin{array}{l}\text { Total } \\
\%\end{array}$ & $\begin{array}{l}\text { No. } \\
21,6\end{array}$ & $\begin{array}{c}19 \\
76,6\end{array}$ & $\begin{array}{l}66 \\
2,2\end{array}$ & 2 \\
\hline
\end{tabular}

\section{TABLA 5}

Valores promedio y desviación estándar de algunas medidas e índices antropométricos según clasificación clínica y sexo. Medidas / Índices
antropométricos

\begin{tabular}{|c|c|}
\hline \multicolumn{2}{|c|}{$\begin{array}{l}\text { asintomático } \\
\qquad(n=42)\end{array}$} \\
\hline Media & DS \\
\hline 27.9 & 3.3 \\
\hline 11.9 & 3.8 \\
\hline 47.5 & 13.3 \\
\hline 15.6 & 5.6 \\
\hline
\end{tabular}

masculino

sida
$(n=24)$
Media

28.2

11.9

3.5

45.5

3.9

16.6
DS

.5
.9
2.6
7.7

6

\begin{tabular}{cccc}
\multicolumn{3}{c}{ asintomático } \\
Media & $\mathrm{n}=12)$ & \multicolumn{2}{c}{$\begin{array}{c}\text { sida } \\
(\mathrm{n}=9)\end{array}$} \\
& DS & Media & DS \\
27.7 & 3.7 & 29.3 & 4.6 \\
12.6 & 5.2 & 12.8 & 4.5 \\
45.5 & 12.6 & $52.1^{*}$ & 15.5 \\
16.6 & 7.7 & 18.0 & 8.2 \\
\hline
\end{tabular}

Fuente: Encuestas.

${ }^{*} p<0,05$ : Diferencia estadísticamente significativa. 
VI). También fue frecuente el huevo (grupo IV).

Como puede observarse en el gráfico 2, algo más de la mitad de los sujetos encuestados $(51,7 \%)$ presentaban un nivel de conocimientos y actitudes considerado suficiente. Las personas asintomáticas presentaban un mayor porcentaje de sujetos con conocimientos y actitudes sobre la enfermedad que los del grupo con sida (35,6 y 16,1\% respectivamente).

\section{DISCUSIÓN}

Como se menciónó, para evaluar el estado de nutrición se utilizaron indicadores bioquímicos en sangre tales como: glicemia, triglicéridos, colesterol, hemoglobina, proteínas totales, transferrinas, entre otros (3).

Las concentraciones séricas normales de triglicéridos durante la fase asintomática de la enfermedad pueden deberse a que los niveles de citoquinas responsables de su síntesis (IL-1, TNF, TNFa) están más bajos que en el estadio sida (10).

Los cambios metabólicos relacionados con los triglicéridos en pacientes con sida, desencadenan un ciclo inútil con los ácidos grasos, los cuales son movilizados del tejido adiposo y llevados al hígado, no para ser oxidados, como normalmente ocurre, sino para ser esterificados y secretados como VLDL

\section{GRÁFICO 1}

Distribución porcentual de la frecuencia de comida según clasificación clínica.

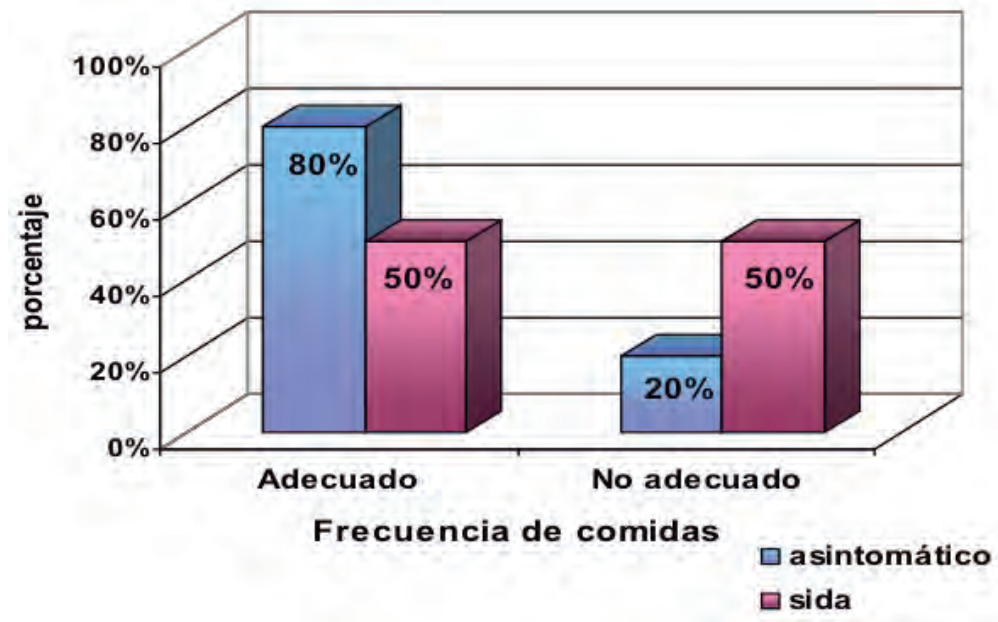

TABLA 6

Distribución porcentual de los grupos de alimentos según frecuencia de consumo y clasificación clínica.

Grupos de alimentos

Cereales y Viandas

Vegetales

Frutas

Carnes rojas

Carnes blancas

Pescados

Huevo

Granos

Lácteos

Grasa vegetal

Azúcares y Dulces
Asintomáticos

Habitual

No Habitual

$5 \%$

$55 \%$

$56 \%$

$48 \%$

$39 \%$

$69 \%$

30\%

$31 \%$

$17 \%$

0

$40 \%$

$70 \%$

$83 \%$ sida

Habitual No Habitual

$91 \%$

$9 \%$

$73 \%$

$27 \%$

$18 \%$

$82 \%$

$21 \%$

$79 \%$

$39 \%$

$61 \%$

$9 \%$

$91 \%$

$52 \%$

$48 \%$

$64 \%$

$36 \%$

$94 \%$

$6 \%$

$100 \%$

0

$15 \%$

Fuente: Encuesta. 
aumentando los niveles plasmáticos de dicha lipoproteína y de su fracción lipídica más abundante: los triglicéridos (11).

Lo anterior permite sugerir que el aumento de los triglicéridos a valores al menos cercanos al límite superior del intervalo normal establecido para adultos sanos, pudiera ser considerado como indicador lipídico asociado con inicio de las manifestaciones clínicas del sida.

La presencia de hipertrigliceridemia e hiperglicemia pudiera hacernos pensar en el desarrollo de la lipodistrofia que puede presentarse en estas personas asociada o no a la terapia antirretroviral.

Se ha reportado que el tratamiento de la infección de VIH con terapia antirretroviral altamente activa puede desencadenar complicaciones metabólicas severas que incluyen lipodistrofia, dislipidemia y resistencia a la insulina, siendo factor de riesgo de enfermedad cardiovascular y diabetes tipo $2(12,13)$.

En las personas con VIH/sida se presenta una disminución importante de la grasa corporal sobre todo en la fase sida $(10,11)$. Es en este estadio donde se requiere fundamentalmente el uso de las reservas energéticas para combatir infecciones oportunistas ya que el paciente sufre con más intensidad procesos infecciosos que afectan las diferentes partes del tubo digestivo, caracterizados por mala absorción y/o diarreas crónicas. Lo anterior unido a la anorexia como factor fundamental provoca disminución en el consumo de alimentos, pérdida de peso y alteraciones del IMC.

Las personas con VIH y tratamiento con antirretrovirales pueden perder grasa subcutánea (lipoatrofia) en ausencia de depleción de la masa muscular, lo que puede confundir la interpretación clínica de la pérdida de peso hasta 10\%, que caracteriza a la lipodistrofia (19).

El valor promedio del Índice de masa corporal en las personas con VIH reportado por algunos autores (15), fue $21,2 \mathrm{Kg} / \mathrm{m}^{2}$, otros autores reportan valores de IMC similares como Sharpstone (16) que refiere $22,4 \mathrm{Kg} / \mathrm{m}^{2}$ y este mismo autor señala lo recogido por otros investigadores tales como Pichard 21,8 Kg/m², Jiménez 20,7 kg/m² y Rabeneck 20,6 Kg/ $\mathrm{m}^{2}$. Brown (17) reportó un valor que fue $24,1 \mathrm{~kg} / \mathrm{m}^{2}$. Autores más recientes (18) reportan valores de 20 a $24 \mathrm{Kg} / \mathrm{m}^{2}$.

Según las tablas de los percentiles de circunferencia braquial en adultos sanos del sexo masculino, los valores encontrados en este estudio pertenecen a percentiles entre el 10 y el 90, considerado normal (9), lo que puede deberse a que aun con la enfermedad, mantienen un buen estado nutricional y la mayoría tiene una evolución igual o menor a cinco años. Se ha demostrado que los tratamientos actuales permiten elevar la calidad de vida de las personas con sida, lo que pudiera explicar estos resultados (19).

El pliegue cutáneo del tríceps en este estudio fue superior a los presentados por Arnalich et al (20) 1997 y por Sicotte et al (21) en 2010. Sin embargo, Sharkey et al (22) en 1992 reportan valores más bajos. Estas diferencias podrían explicarse por las distintas etapas clínicas en las cuales se realizaron esos estudios.

Ortega y Marti (23) encontraron valores de pliegue tricipital sin especificación de sexo entre 15 y 22 mm, cifras superiores a las encontradas en este estudio para el sexo femenino.

Kotler (24) ha hecho énfasis en que el desgaste de los pacientes con sida se caracteriza por una pérdida de la masa celular corporal y no por la pérdida de grasa corporal. Sin embargo, en este estudio se demuestra mayores disminuciones de los indicadores de depósitos grasos que de proteínas. Ellos concluyeron que la pérdida de peso observada en los pacientes con sida se deben principalmente a la depleción de la reserva grasa.

En el análisis de frecuencia de comida en el día se evidencia que los sujetos asintomáticos presentan un predominio de comportamiento adecuado en la frecuencia de consumo de alimentos en el día no así los del grupo sida en que la mitad de ellos presentó comportamiento no adecuado, lo que puede

\section{GRÁFICO 2}

Nivel de conocimientos y actitudes según clasificación clínica.

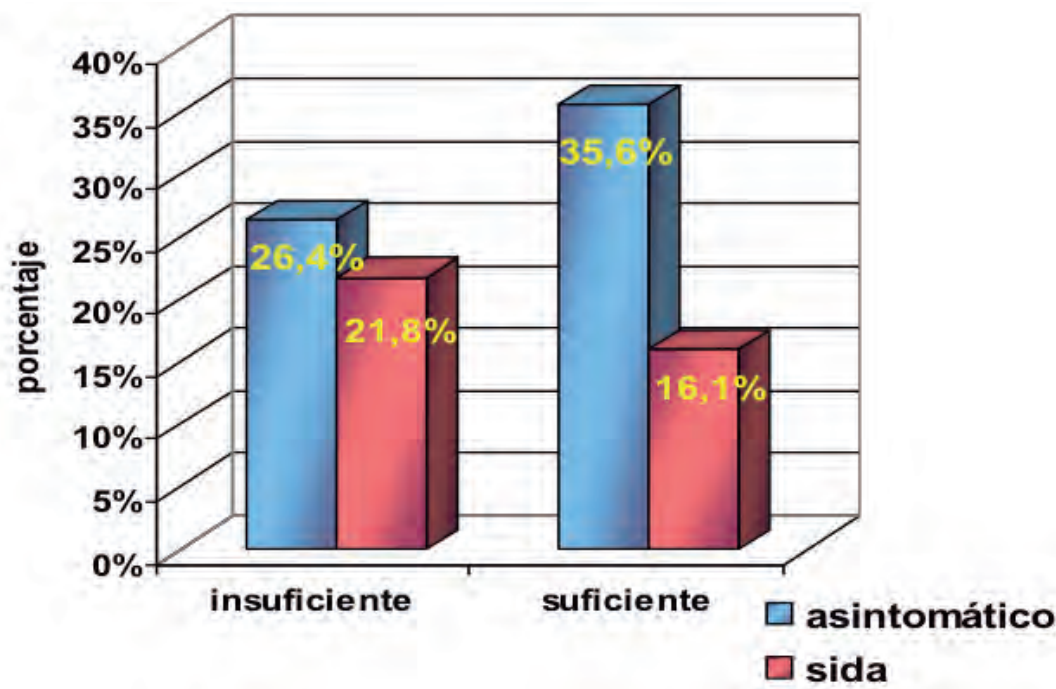


provocar mayores complicaciones de la infección.

Llama la atención la baja frecuencia de consumo de pescados y frutas en las dos categorías, debido a que estos alimentos son excelentes fuentes de sustancias esenciales en el organismo, que tienen función antioxidante y pueden ayudar a palear los radicales libres que se forman por el mismo estrés oxidativo que sufren las personas que viven con el virus de inmunodeficiencia humana.

Al ser diagnosticados se le imparte el curso "Aprendiendo a vivir con el $\mathrm{VIH}$ " que incluyen temas referentes a la alimentación y la nutrición y a todas las personas con VIH/ sida que reciben la dieta 32.24 (establecida por el dietario nacional del MINSAP) se les ofrece la ayuda alimentaria del Fondo de las Naciones Unidas para el desarrollo (PNUD), cuyo objetivo es brindar una alimentación hipercalórica e hiperproteica, y contribuir a mejorar la calidad de vida de las personas infectadas $(5,25)$.

El nivel de conocimientos y actitudes fue considerado suficiente. Estos resultados pueden estar asociados a las campañas publicitarias y sobre todo a los programas educativos que se implementan de forma sistemática en los municipios de salud, organizados por el grupo provincial de VIH-sida, con las personas que recién han sido diagnosticadas (curso "Aprendiendo a vivir con VIH"). En estos programas educativos se dedica parte importante del tiempo a educar a las personas en todo lo relacionado con la nutrición y la estrecha relación de esta con el desarrollo de la enfermedad. La asistencia a dichos cursos es obligatoria (25).

Aunque los conocimientos y actitudes investigados tuvieron un comportamiento adecuado por los resultados de la encuesta de frecuencia, se evidencia la necesidad de incrementar las labores educativas encaminadas a mejorar la actitud de estas personas en relación al cuidado de su nutrición (26-28).

Finalmente se concluye que el estado nutricional de las personas estudiadas no puede considerarse óptimo, pero puede mejorar con una orientación nutricional adecuada.

En el transcurso de este estudio se ha hecho evidente que el uso inteligente de la anamnesis, exploración clínica y antropométrica y la selección de pruebas complementarias adecuadas constituye la forma más eficaz de orientar un trastorno nutricional para poder instaurar pronto medidas terapéuticas y determinar aquellos casos que deben ser remitidos para una evaluación nutricional más completa.

\section{RESUMEN}

Está demostrada la relación entre la alimentación, la nutrición y la infección por VIH. La comunidad científica reconoce que los conocimientos y el cuidado nutricional pueden contribuir a mantener la salud y a disminuir los efectos de la infección. Objetivo: Evaluar el estado nutricional de las personas con VIH-sida atendidas en la consulta de descentralización del municipio Plaza. Sujetos y Métodos: Se realizó un estudio descriptivo transversal en 87 personas infectados con el VIH, 66 del sexo masculino y 21 femenino. 54 portadores asintomáticos del virus y 33 pacientes sida. La evaluación nutricional consistió en mediciones antropométricas: peso, talla, pliegue tricipital, circunferencia braquial para calcular el índice de masa muscular, área grasa y muscular del brazo; evaluación dietética con encuestas de frecuencia de consumo de alimentos y por grupo de alimentos; medición de parámetros bioquímicos: hemoglobina, triglicéridos, glicemia y colesterol. Se comparó con la prueba t de Student así como correlación de Spearman entre indicadores. Resultados: Predominaron los asintomáticos masculinos entre 30-49 años. Se detectaron pocos casos de anemia. Predominó la hipertrigliceridemia y disminuciones discretas de las reservas de grasas. La mitad de personas con sida presentó comportamiento alimentario inadecuado. Más de la mitad presentó nivel de conocimientos y actitud suficiente. Conclusión: Es necesario incrementar la actitud de estos pacientes en relación al cuidado de su nutrición.

Palabras clave: evaluación nutricional, VIH sida, índice de masa corporal, antropometría.

Agradecimientos: A la Dra. Georgina María Zayas Torriente, por sus sabios consejos y su apoyo incondicional a la realización de esta investigación. Al Msc. William Carvajal y la Técnica antropometrista Miriam Martínez por la ayuda brindada en las mediciones antropométricas. Al Dr. Vladimir Reymond González y al Licenciado Onay A. Mercader Camejo, por su estímulo, entusiasmo y compañía en todos los momentos de esta tarea.

\section{BIBLIOGRAFÍA}

1. De Pee S, Semba RD. Role of nutrition in HIV infection: review of evidence for more effective programming in resource-limited settings. Food Nutr Bull. 2010;31(4):S31344.

2. Raiten DJ. Nutrition and pharmacology: general principles and implications for HIV. Am J Clin Nutr. 2011; 94(6): 1697S-702S.

3. Basabe Tuero B, Díaz ME, Martín I, Rodríguez Ojea A. Maestría de nutrición en salud. Evaluación del estado nutricional [CD.ROM]. La Habana: INHA; 2008.

4. Botros D, Somarriba $G$, Neri D, Miller TL. Interventions to address chronic disease and HIV: strategies to promote exercise and nutrition among HIV-infected individuals. Curr HIV/AIDS Rep. 2012;9(4):351-63.

5. Zayas Torriente GM. Nutrición y SIDA. Ministerio de Salud Pública. La Habana. Cuba. 2004.

6. Control Diseases Center. Definition for AIDS among adolescents and adults. MMWK. 1993; 41(17):1-17.

7. OMS WHO/EHT. Review of Haemoglobin Colour Scale: Report of an informal consultation. Review of Studies of the HbCs. Geneva, Switzerland: WHO Headquarters; 2004. p. 3-12.

8. Grundy SM, Cleeman JI, Merz CN, Brewer HB, Clark T. Implications of recent clinical trials for the national cholesterol education program adult treatment panel III guidelines. J Am Coll Cardiol. 2004; 44(3), 720-32.

9. Berdasco A, Romero del Sol JM. Circunferencia del brazo como evaluadora del estado nutricional del adulto. Rev Cubana Aliment y Nutr. 1998; 12(2): 86-90.

10. Hollmann A, Matos PM, Augusto MT, Castanho MA, Santos NC. Conjugation of cholesterol to HIV-1 fusion inhibitor C34 increases peptide-membrane interactions potentiating its action. PLoS One. 2013;8(4):e60302.

11. Cassol E, Misra V, Holman A, Kamat A, Morgello S, Gabuzda D. Plasma metabolomics identifies lipid abnormalities linked to markers of inflammation, microbial translocation, and hepatic function in HIV patients receiving protease inhibitors. BMC Infect Dis. 2013; 4;13:203.

12. Gillard BK, Raya JL, Ruiz-Esponda R, lyer D, Coraza I, Balasubramanyam A. Impaired lipoprotein processing in HIV patients on antiretroviral therapy: aberrant high-density lipoprotein lipids, stability, and function. Arterioscler Thromb Vasc Biol. 2013;33(7):1714-21.

13. Misra R, Chandra P, Riechman SE, Long DM, Balasubramanyam A. Relationship of ethnicity and CD4 Count with 
glucose metabolism among HIV patients on Highly-Active Antiretroviral Therapy (HAART). BMC Endocr Disord. 2013; 22;13(1):13.

14. Souza SJ, Luzia LA, Santos SS, Rondó PH. Lipid profile of HIV-infected patients in relation to antiretroviral therapy: a review. Rev Assoc Med Bras. 2013;59(2):186-98.

15. Evans D, McNamara L, Maskew M, Selibas K, van Amsterdam D, Baines N. Impact of nutritional supplementation on immune response, body mass index and bioelectrical impedance in HIV-positive patients starting antiretroviral therapy. Nutr J. $2013 ; 12(1): 111$.

16. Sharpstone D, Murray C, Ross H, Phelan M, Crane R. The influence of nutritional and metabolic status on progression from asymptomatic HIV infection to AIDS-defining diagnosis. Aids. 1999; 13(10), 1221-6.

17. Brown TT, Chen Y, Currier JS, Ribaudo HJ, Rothenberg J. Body composition, soluble markers of inflammation, and bone mineral density in antiretroviral therapy-naive HIV-1-infected individuals. J Acquir Immune Defic Syndr. 2013;63(3):323-30.

18. Núñez-Rocha GM, Wall KM, Chávez-Peralta M, SalinasMartínez AM, Benavides-Torres RA. Nutritional care, time period since diagnosis, demographics and body mass index in HIV/AIDS patients. Rev Invest Clin. 2013;65(4):291-9.

19. Kiage JN, Heimburger DC, Nyirenda CK, Wellons MF, Kabagambe EK. Cardiometabolic risk factors among HIV patients on antiretroviral therapy. Lipids Health Dis. 2013;12:50.

20. Arnalich F, Martinez P, Hernanz A, González J, Plaza MA, Montiel C, Peña JM, Vázquez JJ. Altered concentrations of appetite regulators may contribute to the development and maintenance of HIV-associated wasting. AIDS. 1997;11(9):1129-34.
21. Sicotte M, Ledoux M, Zunzunegui MV, Ag Aboubacrine S, Nguyen VK; ATARAO group. Reliability of anthropometric measures in a longitudinal cohort of patients initiating ART in West Africa. BMC Med Res Methodol. 2010;10:102.

22. Sharkey SJ, Sharkey KA, Sutherland LR, Church DL. Nutritional status and food intake in human immunodeficiency virus infection. GI/HIV Study Group. J Acquir Immune Defic Syndr. 1992;5(11):1091-8.

23. Ortega-García MP, Marti Bonmatí E. Valoración del estado nutricional y de la absorción intestinal en pacientes asintomáticos infectados por el virus de la inmunodeficiencia humana (VIH) con y sin hepatitis C crónica. Nutr Hosp. 2006; 21(4): 542-6.

24. Kotler HP, Behrman JR, Watkis SC. Social networks and HIV/AIDS risk perceptions. Demography. 2007; 44 (1): 1-33.

25. Cancio Enrique I, Sánchez Fuentes J, Reymond González V, López Rodríguez V. Información básica sobre la infección por VIH-SIDA. En: Información básica sobre la atención integral a personas viviendo con VIH-SIDA. La Habana. Ed. CNP ITS-VIH-SIDA, 2006. p. 45-72.

26. Sarma $H$, Oliveras $E$. Implementing HIV/AIDS education: impact of teachers' training on HIV/AIDS education in Bangladesh. J Health Popul Nutr. 2013;31(1):20-7.

27. Dibari $F$, Bahwere $P$, Huerga $H$, Irena AH, Owino V. Development of a cross-over randomized trial method to determine the acceptability and safety of novel ready-to-use therapeutic foods. Nutrition. 2013;29(1):107-12.

28. Grobler L, Siegfried N, Visser ME, Mahlungulu SS, Volmink J. Nutritional interventions for reducing morbidity and mortality in people with HIV. Cochrane Database Syst Rev. 2013; 28 (2):4536. 